\title{
The Size of Metastasis in the Sentinel Node Is a Predictor of Additional Non-Sentinel Node Positivity
}

\author{
Todd A. Baker ${ }^{1}$, Nitin Wadhwani ${ }^{2}$, Prabha Rajan ${ }^{2}$, Sharfi Sarker ${ }^{1}$, Gerard Aranha ${ }^{1}$, Margo Shoup ${ }^{1}$, \\ Holly Mattix-Kramer ${ }^{3}$, Constantine Godellas ${ }^{1^{*}}$ \\ ${ }^{1}$ Department of Surgery, Stritch School of Medicine, Loyola University Chicago, Maywood, USA; ${ }^{2}$ Department of Pathology, Stritch \\ School of Medicine, Loyola University Chicago, Maywood, USA; ${ }^{3}$ Department of Preventative Medicine and Epidemiology, Stritch \\ School of Medicine, Loyola University Chicago, Maywood, USA. \\ Email: "cgodellas@lumc.edu
}

Received August $29^{\text {th }}, 2012$; revised September $30^{\text {th }}, 2012$; accepted October $8^{\text {th }}, 2012$

\begin{abstract}
Background: The need for axillary lymph node dissection (ALND) when sentinel lymph nodes (SLN) contain micrometastasis is controversial. The purpose of this study was to determine if the size of tumor in the SLN corresponds with additional positive non-sentinel lymph nodes (non-SLN) in pT1 breast cancer. Methods: This retrospective review of 483 patients with pT1 breast cancer identified 96 patients with tumor positive SLN biopsies between June 1999 and February 2010. The size of SLN metastasis and the number of tumor positive non-SLN were recorded using AJCC criteria. Receiver operating characteristic analysis was used to discriminate the SLN size with the optimal sensitivity, specificity and likelihood ratios (LR) for additional positive non-SLN. Results: Among 96 patients with a tumor positive SLN, 41\% $(\mathrm{n}=39)$ had micrometastasis, and $59 \%(\mathrm{n}=57)$ had macrometastasis. A positive non-SLN was identified after ALND among 18\% ( $=7$ of 39$)$ with micrometastasis compared with $39 \%(n=22$ of 57$)$ with macrometastasis $(p=0.04)$. The size of the SLN metastasis and presence of additional tumor positive non-SLNs corresponds to a positive likelihood ratio of 1.1 for micrometastasis and 1.6 for macrometastasis $(95 \%$ CI: $0.56-0.74)$. Conclusions: Increased size of tumor in SLN is associated with greater likelihood of non-SLN positivity and should be considered for more aggressive follow-up and therapy.
\end{abstract}

Keywords: Sentinel Node; Axillary Lymph Node Dissection (ALND)

\section{Introduction}

Sentinel lymph node (SLN) biopsy is the standard method for assessment of nodal involvement in clinically negative early breast lesions whereby only SLNs containing metastasis require subsequent axillary lymph node dissection (ALND) [1]. Identification of the SLN allows greater scrutiny of lymphatic tissue most likely to contain metastasis via serial sectioning and immunohistochemical (IHC) analysis, improving the identification of small tumor foci [2]. Improved pathologic methodology has resulted in a greater than seven fold increase in the diagnosis of sentinel node micrometastasis (SNMM) over the last decade [3]. To prevent stage migration, the classification of "isolated tumor cells" was introduced as separate category distinct from micrometastasis [4]. Isolated tumor cells (ITC) are classified as node negative for treatment and prognostic purposes whereas SNMM are considered positive and may necessitate additional axillary surgery.

"Corresponding author.
Official guidelines from the American Society of Clinical Oncology (ASCO) released in 2005 advise all patients with SLN micrometastasis to undergo completion ALND, however, data from the National Cancer Institute's Surveillance Epidemiology and End Results database suggest that fewer than $60 \%$ of patients with SNMM undergo additional axillary nodal clearance $[1,3]$. This change in clinicians' attitude towards small volume SLN metastasis is reflected in a 2009 ASCO survey where $98.5 \%$ of those surveyed regard SNMM as important-however only $23 \%$ of surgeons, $23 \%$ of medical oncologists and $15 \%$ of radiation oncologists recommend ALND for micrometastasis [5]. Although the prognostic value of SNMM remains unclear, the extent of axillary lymph involvement remains important for staging and postoperative management. Since the introduction of SLN biopsy, a proliferation of retrospective studies and predictive models have emerged attempting to predict the occurrence of additional non-sentinel lymph nodes (non-SLN) containing tumor. The extent of SLN tumor metastasis was previously associated with further axillary 
nodal positivity; the majority of studies are pooled cohorts of early and advanced lesions combined in a single analysis. Patients with small tumors and limited nodal involvement may be ideal candidates for more limited axillary interventions. With this in mind, we hypothesized that size of SLN metastasis among early pathologic T1 (pT1) cases will accurately predict additional nonSLN positivity and may be useful in differentiating which subgroups would benefit from more aggressive axillary clearance strategies.

\section{Materials and Methods}

Patients and Tumor Characteristics. After approval of the institutional review board for human research subjects, a retrospective review of a prospectively collected database identified patients who underwent sentinel node biopsy for pT1 invasive breast cancer at Loyola University Medical Center between June 1999 and February 2010. Patients with primary tumors larger than $2 \mathrm{~cm}$ or with pathology limited to ductal carcinoma in-situ were excluded.

Electronic medical records were reviewed for the following patient characteristics: age, sex, extent of breast excision, size of primary tumor, Nottingham tumor grade, tumor sub-classification, estrogen and progesterone receptor status, number of sentinel nodes (SN) removed, frequency of SN containing tumor, size of SN metastasis, presence of SN extranodal tumor extension, number of non-SLNs removed, frequency of non-SLNs containing tumor and peritumoral lymphovascular invasion.

Evaluation of Lymph Nodes. Sentinel nodes were identified by preoperative injection of ${ }^{99 \mathrm{~m}}$ Technetium labeled sulfur colloid and intradermal subareolar injection of vital blue dye (isosulfan or methylene blue). Sentinel nodes were intraoperatively identified by visual blue staining and using a hand held gamma counter. Subclassification of SN size was according to AJCC Cancer Staging Manual $7^{\text {th }}$ ed.: small cell clusters of tumors up to $0.2 \mathrm{~mm}$ or nonconfluent cells containing less than 200 cells per section were classified as isolated tumor cells (ITC), metastasis greater than $0.2 \mathrm{~mm}$ and less than or equal to $2.0 \mathrm{~mm}$ as micrometastasis, and nodal involvement greater than $2.0 \mathrm{~mm}$ as macrometastasis [6,7]. Sentinel nodes identified as ITC or micrometastasis were independently confirmed by a single breast-trained pathologist (P.R.).

The protocol for sectioning and evaluation of sentinel nodes has been previously described. In brief, a $4 \mu \mathrm{m}$ section from sentinel node is taken for frozen section and for permanent section the SN specimen is fixed in $10 \%$ formalin, embedded in paraffin and stained with conventional and eosin stain (H \& E). Permanent sections that do not show metastasis with $\mathrm{H} \& \mathrm{E}$ are serially sectioned for three additional H\&E staining and one immunohisto- chemical (IHC) staining using a pankeratin antibody. Up to four $\mathrm{H} \& \mathrm{E}$ sections and one IHC section are evaluated before reporting a SLN as negative; any foci of metastasis is measured and classified according to AJCC guidelines. When multiple foci of tumor are present the largest width is used for staging purposes. Tumor histologic grade was assessed using the Nottingham grading system [8].

Statistical Analysis. Continuous variables were described as medians and interquartile ranges (25th - 75th percentile). Normal distribution was assessed with the D'Agostino-Pearson omnibus K2 test; because not all data sets passed the normality test $(\alpha=0.05)$, the Mann-Whitney U test was used for continuous variables. Fischer's exact test and Pearson's chi-square test were used for dichotomous categorical variables. A two-tailed $\mathrm{p}<0.05$ was considered significant. Receiver operating characteristic (ROC) curves were created to determine the SLN size with the optimal sensitivity and specificity for additional non-SLN positivity. Sensitivity and specificity were used to calculate the positive likelihood ratio (+LR) for additional non-SLN positivity $(+\mathrm{LR}=$ sensitivity/1-specificity $=$ true positives/false positives). The + LR estimates how a positive test result (SLN exceeds a particular threshold) changes the odds of having additional positive non-SLNs. The higher the $+\mathrm{LR}$, the higher the increase in odds of disease given a positive test. Statistical analyses were calculated with Stata/IC 11.0 for Mac OS X (StataCorp) and GraphPad Prism 5 for Mac OS X (GraphPad Software Inc.).

\section{Results}

A total of 483 patients with pT1 invasive breast cancer underwent sentinel node biopsy and resection of the associated primary malignancy with either breast conserving surgery or mastectomy. Of this group, a positive SLN was identified in 96 cases $(19.9 \%)$, consisting of $40.6 \%$ (39/96) micrometastasis and 59.4\% (57/96) macrometastasis. An additional 8 cases of ITC (7.7\%) were observed; these SLN were not classified as a positive SLN biopsy and were included only in the ROC curve analysis as the lowest cut point. Patient demographics and lymph node features are shown in Table 1. Significant differences were noted in method of pathologic detection among groups. The majority of macrometastasis were seen on $\mathrm{H}$ $\& \mathrm{E}$ whereas micrometastasis were predominantly identified using immunohistochemistry. Sentinel node macrometastasis were significantly more likely to demonstrate extranodal tumor extension. As illustrated in Figure 1, sentinel nodes containing macrometastasis had a greater frequency of tumor positive non-SLN $(38.6 \%$ 22/57) when compared to SLN containing micrometastasis $(17.9 \%, 7 / 39, p=0.04)$. The number of total positive non-SLN in each instance was significantly greater for SLN containing macrometastasis (Table $1, p=0.02$ ). 
Table 1. Patient data and lymph node features among patients with positive sentinel lymph nodes.

\begin{tabular}{|c|c|c|c|}
\hline & $\begin{array}{l}\text { Micrometastasis } \\
\qquad \mathrm{N}=39(\%)\end{array}$ & $\begin{array}{l}\text { Macrometastasis } \\
\qquad \mathrm{N}=57(\%)\end{array}$ & p value \\
\hline Median age, yrs (IQR) & $58(47-71)$ & $56(47-67)$ & 0.71 \\
\hline Age $<50$ years & $14(35.9)$ & $17(29.8)$ & 0.27 \\
\hline Age $>70$ years & $9(23.1)$ & $11(19.3 \%)$ & 0.80 \\
\hline \multicolumn{4}{|l|}{ Procedure } \\
\hline Partial mastectomy & $28(71.8)$ & $39(68.4)$ & 0.82 \\
\hline Mastectomy & $11(28.2)$ & $18(31.6)$ & 0.72 \\
\hline \multicolumn{4}{|l|}{ Sentinel lymph nodes } \\
\hline $\begin{array}{l}\text { Median no. SLN } \\
\text { removed, (IQR) }\end{array}$ & $2(1-3)$ & $2(1-3)$ & 0.25 \\
\hline $\begin{array}{l}\text { Median size SLN } \\
\text { metastasis, cm (IQR) }\end{array}$ & $0.14(0.1-0.2)$ & $0.7(0.25-1.1)$ & $<0.0001^{*}$ \\
\hline $\begin{array}{l}\text { Detection by } \mathrm{H} \& \mathrm{E} \\
\text { stain }\end{array}$ & $24(61.5)$ & $52(91.2)$ & $0.0007^{*}$ \\
\hline $\begin{array}{l}\text { Detection by pankeratin } \\
\text { IHC }\end{array}$ & $23(59.0)$ & $7(12.3)$ & $<0.0001^{*}$ \\
\hline $\begin{array}{l}\text { Extrahilar nodal } \\
\text { extenstion }\end{array}$ & $1(2.6)$ & $23(40.4)$ & $<0.0001^{*}$ \\
\hline \multicolumn{4}{|l|}{$\begin{array}{l}\text { Axillary lymph node } \\
\text { dissection }\end{array}$} \\
\hline $\begin{array}{l}\text { Median no. non-SLN } \\
\text { removed, (IQR) }\end{array}$ & $11(2-17)$ & $14(10-18)$ & 0.06 \\
\hline $\begin{array}{l}\text { Median no. non-SLN } \\
\text { with tumor, (IQR) }\end{array}$ & $1(1-3)$ & $2(1-5)$ & $0.02^{*}$ \\
\hline $\begin{array}{l}\text { Freq. non-SLN positve } \\
\text { on ALND }\end{array}$ & $7(17.9)$ & $22(38.6)$ & $0.04^{*}$ \\
\hline
\end{tabular}

*statistically significant $\mathrm{p}<0.05$; IQR: Interquartile Range $(25$ th -75 th percentile); SLN: Sentinel Lymph Nodes; H \& E: Hematoxylin \& Eosin; IHC: Immunohistochemistry; ALND: Axillary Lymph Node Dissection.

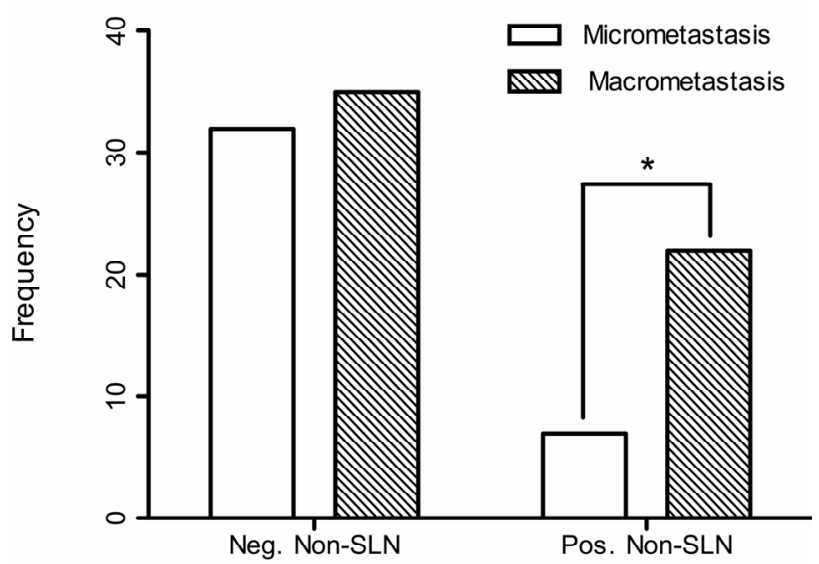

Figure 1. Frequency of non-sentinel lymph nodes (non-SLN) containing metastasis stratified according to the size of tumor in the sentinel node biopsy. Additional positive nonSLNs identified in SLN with micrometastasis $17.9 \%$ (7/39) versus $38.6 \%(22 / 57)$ in SLN containing macrometastasis ( $\left.{ }^{*} \mathrm{p}=\mathbf{0 . 0 4}\right)$.
Features of the primary tumors in both subsets were also compared as shown in Table 2. Among the 96 patients with a positive SLN biopsy there was no difference in breast pathology between groups. The median size of the primary tumor was significantly larger among cases of macrometastasis. No significant differences were observed between classifications of SLN metastasis for Nottingham grade, estrogen and progesterone receptor status, as well as peritumoral lymphovascular invasion.

The results of Table 3 and Figure 2 summarize the association between increasing SLN tumor size and the likelihood of an additional positive non-SLN. The positive likelihood ratio for additional non-SLN metastasis is approximately 1.1 for micrometastasis and 1.6 in the case of micrometastasis $(95 \%$ CI $0.55-0.74)$. The ROC curve shows that increasing the size of SLN metastasis improves the specificity for identifying positive non-SLN while maintaining sensitivity corresponding to an area under the curve (AUC) of 0.6502 .

\section{Discussion}

In the present study we demonstrate an association between the size of metastasis in the SLN and the presence of additional positive non-SLN in pT1 invasive breast cancer. Both SLN micro and macrometastasis have an increased likelihood of additional positive non-SLNs.

Table 2. Features of primary tumors with sentinel nodes containing metastasis.

\begin{tabular}{|c|c|c|c|}
\hline & Micrometastasis & Macrometastasis & $p$ value \\
\hline & $\mathrm{N}=39(\%)$ & $\mathrm{N}=57(\%)$ & \\
\hline \multicolumn{4}{|l|}{ Primary tumor } \\
\hline Median size, cm (IQR) & $1.2(0.7-1.5)$ & $1.4(0.1-2.0)$ & $0.01^{*}$ \\
\hline $\begin{array}{l}\text { Median Nottingham } \\
\text { grade, (gr. } 1,2,3 \text { ) }\end{array}$ & $2(1-2)$ & $2(1-2)$ & 0.51 \\
\hline \multicolumn{4}{|l|}{ Pathology } \\
\hline Infiltrating ductal & $29(74.4)$ & $40(70.2)$ & 0.82 \\
\hline Infiltrating lobular & $6(15.4)$ & $9(15.8)$ & 0.95 \\
\hline Other & $4(10.3)$ & $8(14.0)$ & 0.58 \\
\hline \multicolumn{4}{|l|}{ Receptor status } \\
\hline $\mathrm{ER}+/ \mathrm{PR}+$ & $30(76.9)$ & $42(73.7)$ & 0.81 \\
\hline $\mathrm{ER}+/ \mathrm{PR}-$ & $1(2.6)$ & $7(12.3)$ & 0.14 \\
\hline $\mathrm{ER}-/ \mathrm{PR}+$ & $3(7.7)$ & $2(3.5)$ & 0.39 \\
\hline $\mathrm{ER}-/ \mathrm{PR}-$ & $5(12.8)$ & $6(10.5)$ & 0.75 \\
\hline $\begin{array}{l}\text { Lymphovascular } \\
\text { invasion, no. (\%) }\end{array}$ & $10(25.6)$ & $17(29.8)$ & 0.82 \\
\hline
\end{tabular}


Table 3. Receiver operating characteristic analysis of nonsentinel lymph nodes.

\begin{tabular}{lccccc}
\hline \multicolumn{5}{c}{ Status of Non-SLN } & \\
\hline $\begin{array}{l}\text { Size SLN } \\
\text { Metastasis }\end{array}$ & Negative & Positive Sensitivity & Specificity & + L.R. \\
\hline $\begin{array}{l}\text { Isolated Tumor } \\
\text { Cells }\end{array}$ & 8 & 0 & $100 \%$ & $0 \%$ & 1.00 \\
Micrometastasis & 7 & 32 & $100 \%$ & $10.0 \%$ & 1.13 \\
Macrometastasis & 22 & 35 & $75.9 \%$ & $51.4 \%$ & 1.56 \\
ROC Area & 0.6502 & & & & \\
Standard Error & 0.048 & & & & \\
$95 \%$ CI & $0.56-0.74^{*}$ & & & & \\
\hline
\end{tabular}

*statistically significant; ROC: Receiver operating characteristic; Non-SLN: Non-Sentinel Lymph Nodes.

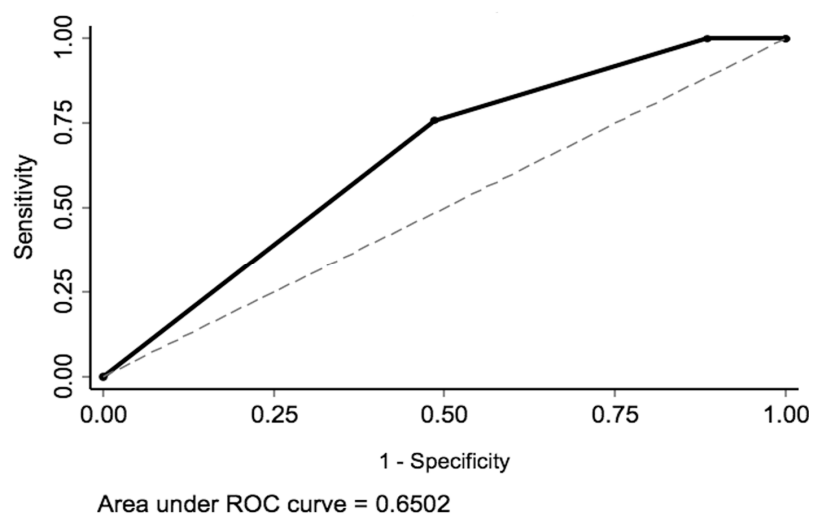

Figure 2. Nonparametric receiver operating characteristic curve identifies the threshold of a positive screening test that optimizes sensitivity and specificity as expressed by the area under curve (AUC). Increasing the size of SLN metastasis improves the specificity for identifying positive nonSLN with an AUC = 0.6502 (S.E. 0.048. 95\% CI 0.56 - 074).

Increasing the size of tumor in SLNs corresponds to a greater than two fold increase in the prevalence of non-SLN metastasis, from $17.9 \%$ among micrometastasis to $38.6 \%$ with macrometastasis. A positive SLN biopsy will increase the odds of additional non-SLN metastasis by a factor of 1.1 in micrometastasis and by nearly 1.6 when macrometastasis is present.

The size of the primary tumor within the pT1 subclass was also associated with non-SLNs containing metastasis. Extranodal tumor extension found predominantly in SLN macrometastasis was also associated with positive nonSLNs on univariate analysis. The association between extranodal tumor extension, macrometastasis and axillary nodal dissemination is demonstrative of the increased metastatic potential of larger neoplasms. Previous studies have shown more aggressive patterns of tumor spread when peritumoral lymphovascular invasion is present $[9,10]$. The present study did not find differences in the occurrence of peritumoral lymphovascular invasion nor a tendency for nodal spread among cases of SLN micro and macrometastasis.

Both the size of the primary lesion and the extent of regional metastasis are mutually reflective of the tumor's growth potential. Turner, et al identified a similar increase in non-SLN positivity among pT1-T4 lesions with larger primary tumors and increasing SLN tumor burden. In this study, SLN micrometastasis corresponded to a $26 \%$ incidence of non-sentinel nodal positivity and a $63 \%$ occurrence for SLN containing macrometastasis [9]. In other case series limited to pT1-T2 neoplasms, the frequency of non-SLN positivity ranges from $6 \%-22 \%$ for SLN with micrometastasis and 44\% - 55\% for macrometastasis [10-12]. In a meta-analysis of 25 articles reporting non-SLN spread concurrent with small volume SLN metastasis, Cserni, et al report a risk of additional axillary disease ranging from $10 \%-15 \%$ [13]. The trend towards decreased incidence of non-SLN metastasis corresponding to smaller primary tumors was also reflected in the present study.

There are several explanations for the variability that is noted among retrospective reviews attempting to quantify and correlate sizing of SLN metastasis. Studies were routine ALND was performed regardless of SLN status may have higher estimates of non-SLN positivity compared to those study protocols allowing omission of surgical axillary clearance. [13] side from selection biases, the method of pathologic detection will affect the sensitivity-specifically the utilization of $\mathrm{H} \& \mathrm{E}$ versus IHC. As noted in the present study, $\mathrm{H} \& \mathrm{E}$ was more often utilized for identifying macrometastasis whereas pankeratin IHC was more frequently utilized for micrometastasis.

Although the association between small volume SLN metastasis and additional non-SLN is clearly applicable to early pT1 tumors, the clinical significance is less certain. Official guidelines from an ASCO panel of experts continue to recommend ALND in the presence of micro and macrometastasis on SLN biopsy. This has been called into question by the recent publication from the ACOSOG Z0011 trial showing no difference in local or regional recurrence among patients with limited disease randomized to SLND alone versus SLND followed by ALND [14]. Participants of this study received lumpectomy and opposing tangential whole field radiation for T1 or T2 N0 M0 disease. Standard opposing tangential field radiation is known to concurrently irradiate portions of the level I and II axillary lymph nodes and may have an effect on axillary recurrence notwithstanding the differences in surgical axillary clearance $[15,16]$. The number of positive axillary lymph nodes remains a relevant risk factor for local recurrence among patients undergoing mastectomy without radiotherapy [17]. The effect of ACOSOG Z0011 trial is further confounded by the ad- 
ministration of adjuvant systemic therapy known to reduce locoregional recurrence. Patients who do not receive radiation and adjuvant systemic therapy may not achieve the same results as in the Z0011 trial and may still benefit from additional scrutiny of their axillary nodal status.

\section{Conclusion}

The present study demonstrates a significant correlation between the size of tumor in the SLN and axillary metastasis to non-SLN in patients with pT1 breast cancer. The size of the SLN metastasis is an important risk factor even among early breast lesions. Although the strength of association is strongest for patients with macrometastasis, our data suggests that patients with either micro or macrometastasis in their SLN biopsies have an increased likelihood of additional nodal positivity. This has important therapeutic implications given the recent trend towards minimizing axillary interventions in perceived low risk subpopulations. The findings of this study suggest patients with pT1 lesions and large volumes of SLN tumor should be considered for closer follow-up and more aggressive axillary management strategies.

\section{REFERENCES}

[1] G. H. Lyman, A. E. Giuliano, M. R. Somerfield, A. B. Benson, D. C. Bodurka, H. J. Burstein, A. J. Cochran, et al., "American Society of Clinical Oncology Guideline Recommendations for Sentinel Lymph Node Biopsy in Early-Stage Breast Cancer," Journal of Clinical Oncology, Vol. 23, No. 30, 2005, pp. 7703-7720. doi:10.1200/JCO.2005.08.001

[2] A. E. Giuliano, P. S. Dale, R. R. Turner, D. L. Morton, S. W. Evans and D. L. Krasne, "Improved Axillary Staging of Breast Cancer with Sentinel Lymphadenectomy," Annals of Surgery, Vol. 222, No. 3, 1995, pp. 399-401. doi:10.1097/00000658-199509000-00016

[3] N. Wasif, M. A. Maggard, C. Y. Ko and A. E. Giuliano, "Underuse of Axillary Dissection for the Management of Sentinel Node Micrometastases in Breast Cancer," Archives of Surgery, Vol. 145, No. 2, 2010, pp. 161-166. doi:10.1001/archsurg.2009.269

[4] D. L. Weaver, "Sentinel Lymph Nodes and Breast Carcinoma: Which Micrometastases Are Clinically Significant?" The American Journal of Surgical Pathology, Vol. 27, No. 6, 2003, pp. 842-845. doi:10.1097/00000478-200306000-00018

[5] N. Wasif, X. Ye and A. E. Giuliano, "Survey of ASCO Members on Management of Sentinel Node Micrometastases in Breast Cancer: Variation in Treatment Recommendations According to Specialty," Annals of Surgical Oncology, Vol. 16, No. 9, 2009, pp. 2442-2449. doi:10.1245/s10434-009-0549-7

[6] S. D. Edge, D. R. Byrd, C. C. Compton, A. G. Fritz, F. L. Greene, A. Trotti, et al., "AJCC Cancer Staging Hand- book, from the AJCC Cancer Staging Manual," 7th Edition, Springer, New York, 2009.

[7] M. Hulvat, P. Rajan, E. Rajan, S. Sarker, C. Schermer, G. Aranha, et al., "Histopathologic Characteristics of the Primary Tumor in Breast Cancer Patients with Isolated Tumor Cells of the Sentinel Node," Surgery, Vol. 144, No. 4, 2008, pp. 518-524. doi:10.1016/j.surg.2008.06.006

[8] C. W. Elston, "The Assessment of Histological Differentiation in Breast Cancer," Australian and New Zealand Journal of Surgery, Vol. 54, No. 1, 1984, pp. 11-15. doi:10.1111/j.1445-2197.1984.tb06677.x

[9] R. R. Turner, K. U. Chu, K. Qi, L. E. Botnick, N. M. Hansen, E. C. Glass and A. E. Giuliano, "Pathologic Features Associated with Nonsentinel Lymph Node Metastases in Patients with Metastatic Breast Carcinoma in a Sentinel Lymph Node," Cancer, Vol. 89, No. 3, 2000, pp. 574-581. doi:10.1002/1097-0142(20000801)89:3<574::AID-CNCR 12>3.0.CO;2-Y

[10] M. R Weiser, L. L. Montgomery, L. K. Tan, B. Susnik, D. Y. Leung, P. I. Borgen, et al., "Lymphovascular Invasion Enhances the Prediction of Non-Sentinel Node Metastases in Breast Cancer Patients with Positive Sentinel Nodes," Annals of Surgical Oncology, Vol. 8, No. 2, 2001, pp. 145-149. doi:10.1007/s10434-001-0145-y

[11] K. U. Chu, R. R. Turner, N. M. Hansen, M. B. Brennan, A. Bilchik and A. E. Giuliano, "Do All Patients with Sentinel Node Metastasis from Breast Carcinoma Need Complete Axillary Node Dissection?" Annals of Surgery, Vol. 229, No. 4, 1999, pp. 536-541. doi:10.1097/00000658-199904000-00013

[12] G. Viale, E. Maiorano, G. Mazzarol, S. Zurrida, V. Galimberti, A. Luini, et al., "Histologic Detection and Clinical Implications of Micrometastases in Axillary Sentinel Lymph Nodes for Patients with Breast Carcinoma," Cancer, Vol. 92, No. 6, 2001, pp. 1378-1384. doi:10.1002/1097-0142(20010915)92:6<1378::AID-CNC R1460>3.0.CO;2-Y

[13] G. Cserni, D. Gregori, F. Merletti, A. Sapino, M. P. Mano, A. Ponti, et al., "Meta-Analysis of Non-Sentinel Node Metastases Associated with Micrometastatic Sentinel Nodes in Breast Cancer," British Journal of Surgery, Vol. 91, No. 10, 2004, pp. 1245-1252. doi:10.1002/bjs.4725

[14] A. E. Giuliano, L. Mccall, P. Beitsch, P. Whitworth, P. Blumencranz, A. Leitch, et al., "Locoregional Recurrence after Sentinel Lymph Node Dissection with or Without Axillary Dissection in Patients with Sentinel Lymph Node Metastases," Annals of Surgery, Vol. 252, No. 3, 2010, pp. 426-433.

[15] C. Aristei, F. Chionne, A. R. Marsella, M. Alessandro, A. Rulli, A. Lemmi, et al., "Evaluation of Level I and II Axillary Nodes Included in the Standard Breast Tangential Fields and Calculation of the Administered Dose, Results of a Prospective Study," International Journal of Radiation Oncology, Biology and Physics, Vol. 51, No. 1, 2001, pp. 69-73. doi:10.1016/S0360-3016(01)01595-4

[16] P. J. Schlembach, T. A. Buchholz, M. I. Ross, S. M. Kirsner, G. J. Salas, E. A. Strom, et al., "Relationship of Sentinel and Axillary Level I-II Lymph Nodes to Tangential Fields Used in Breast Irradiation," International Jour- 
nal of Radiation Oncology, Biology and Physics, Vol. 51, No. 3, 2001, pp. 671-678.

doi:10.1016/S0360-3016(01)01684-4

[17] J. S. Wong and J. R. Harris, "Postmastectomy Radiation
Therapy,” In: J. R. Harris, M. E. Lippman, M. Morrow and C. K. Osborne, Eds., Diseases of the Breast, 3rd Edition, Lippincott Williams \& Wilkins, Philadelphia, 2004, pp. 785-799. 